\title{
Pulgar en gatillo, pulgar en resorte, o tenosinovitis estenosante del pulgar pediátrico
}

\section{Trigger thumb, spring thumb, or stenosing tenosynovitis pediatric thumb}

Alfonso Migoya-Nuño, ${ }^{1}$ Gabriel Fraind-Maya, ${ }^{2}$ Luis Eduardo Loyo-Soriano

El pulgar en gatillo es una enfermedad idiopática, en la que el tendón flexor largo del pulgar se encuentra engrosado. ${ }^{1,2,3,4}$ Recibe su nombre debido a la alteración funcional de la articulación interfalángica del pulgar, que característicamente aparece con bloqueo en flexión.

La flexión del pulgar depende del correcto deslizamiento del flexor largo del pulgar a través de un sistema de poleas (A1, polea oblicua y A2); en esta alteración el tendón se encuentra engrosado proximal a la polea $\mathrm{A} 1$, generando una discrepancia de tamaño, que impide el deslizamiento del tendón a través de la polea; ${ }^{5}$ de forma característica, el engrosamiento del tendón forma el Ilamado nódulo de Notta..$^{6,7,3}$ Figura 1

De manera global, afecta a 3 de cada 1000 recién nacidos vivos,,$^{5,8,2}$ es más común en la población hispana; ${ }^{5}$ hasta la fecha no existen datos concretos de incidencia en México. Se ha demostrado que es una alteración del desarrollo, ${ }^{9,2,1}$ porque al nacimiento el tendón es de tamaño normal, pero en los primeros 3 años de vida se va engrosando. ${ }^{10,11,9} \mathrm{En}$ 25 a 30\% de los casos ocurre afectación bilateral, ${ }^{2}$ aunque no suele manifestarse al mismo tiempo. ${ }^{5}$ Existen reportes de familias que muestran un patrón de herencia dominante con penetrancia variable, ${ }^{7}$ pero no existe predisposición por género; a diferencia del resto de los dedos, el pulgar en gatillo no se relaciona con alteraciones sistémicas. ${ }^{1,12}$

La manifestación clínica más común aparece en niños de 2 años, ${ }^{1}$ con episodios de "engatillamiento" o bloqueo, que siempre son asintomáticos y en los que existe limitación para la extensión, que progresa hasta generar bloqueo fijo del pulgar en flexión, en la articulación interfalángica, algunas veces acompañada de molestias o dolor al tratar de realizar la extensión asistida del pulgar. ${ }^{1}$ De manera característica puede palparse el engrosamiento del tendón, formando el nódulo de Notta ${ }^{12,13,14}$ en la región volar de la articulación metacarpofalángica.

\footnotetext{
${ }^{1}$ Hospital Ángeles Pedregal, Ciudad de México.

${ }^{2}$ Departamento de Ortopedia y Traumatología, Hospital Ángeles Lomas, Ciudad de México.

${ }^{3}$ Instituto Nacional de Rehabilitación, Luis Guillermo Ibarra Ibarra, Ciudad de México.

Recibido: 10 de diciembre 2020

Aceptado: 10 de febrero 2021

Correspondencia Alfonso Migoya Nuño alfonsomigoya@gmail.com

Este artículo debe citarse como: Migoya Nuño A, Fraind Maya G, LoyoSoriano LE. Pulgar en gatillo, pulgar en resorte, o tenosinovitis estenosante del pulgar pediátrico. Acta Pediatr Méx 2021; 42 (2): 89-91.

DOI: http://dx.doi.org/10.18233/APM42No2pp89-912221
} 


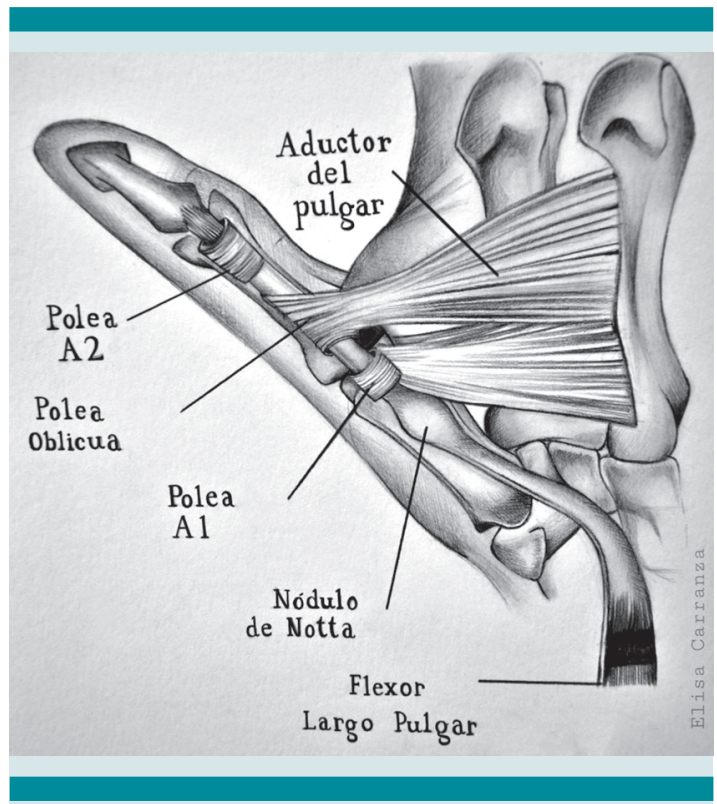

Figura 1. Esquema representativo de la relación anatómica entre el flexor largo del pulgar y la polea A1 del pulgar, se observa un nódulo de Notta.

Durante la exploración física se observan los movimientos activos (realizados por el paciente) y se valora el grado de extensión en relación con el dedo contralateral; también se evalúan los movimientos pasivos (realizados por el médico), donde puede percibirse un clic o resalte en la articulación. ${ }^{14}$ El diagnóstico es clínico, por lo que no están indicadas las radiografías, ${ }^{4,5}$ pues no muestran ninguna alteración y solo se requieren cuando se establece el diagnóstico diferencial con alguna malformación congénita del pulgar.

El diagnóstico diferencial se establece con pulgar flexo aducto congénito, artrogriposis, parálisis cerebral, fracturas y luxaciones de la articulación interfalángica. ${ }^{1,2,5}$

El tratamiento está determinado por la edad; entre $10-30 \%$ de los pacientes menores de 2 años puede curarse de forma espontánea, ${ }^{15,16,2}$ incluso aumenta este valor a $60 \%$ si realizan ejercicios de extensión pasiva por los padres y la colocación de férulas en extensión. ${ }^{6}$ En pacien- tes mayores de 2 años o con contractura fija en flexión se prefiere el tratamiento quirúrgico, pues ha demostrado excelentes resultados y pocas complicaciones, entre las que destacan la lesión del nervio digital y las infecciones. ${ }^{8}$

La cirugía consiste en realizar un acceso transverso de $1 \mathrm{~cm}$ sobre el pliegue palmar de la articulación metacarpofalángica y un corte longitudinal en la polea $\mathrm{A} 1$; no es recomendable efectuar liberaciones percutáneas, porque se asocian con índice de falla de $30 \%$ al no liberar por completo la polea y lesión parcial del tendón en $80 \%$ de los casos. ${ }^{10}$

La referencia al ortopedista pediatra debe hacerse ante cualquier sospecha o al establecer el diagnóstico, pero la mayoría de los pacientes se beneficia del tratamiento quirúrgico.

\section{REFERENCIAS}

1. Shah AS, Bae DS. Management of pediatric trigger thumb and trigger finger. J Am Acad Orthop Surg. 2012;20(4):20613.

2. Twu J, Angeles J. Developmental trigger thumb. Pediatr Ann. 2016;45(4):e135-8.

3. Murgai RR, Lightdale-Miric N. Pediatric trigger thumb caused by a flexor tendon sheath ganglion. J Pediatr Orthop Part B. 2020;29(2):203-5.

4. Giugale JM, Fowler JR. Trigger Finger: Adult and Pediatric Treatment Strategies. Orthop Clin North Am [Internet]. 2015;46(4):561-9. Available from: http://dx.doi. org/10.1016/j.ocl.2015.06.014

5. Bauer AS, Bae DS. Pediatric trigger digits. J Hand Surg Am [Internet]. 2015;40(11):2304-9. Available from: http:// dx.doi.org/10.1016/j.jhsa.2015.04.041

6. Thomas BP, Pallapati S. Congenital thumb differences- current concepts. J Clin Orthop Trauma [Internet]. 2020;11(4):580-9. Available from: https://doi. org/10.1016/j.jcot.2020.06.018

7. Ogino T. Trigger Thumb in Children: Current Recommendations for Treatment. J Hand Surg Am. 2008;33(6):982-4.

8. Farr S, Grill F, Ganger R, Girsch W. Open surgery versus nonoperative treatments for paediatric trigger thumb: A systematic review. J Hand Surg Eur Vol. 2014;39(7):719-26.

9. Verma M, Craig CL, Dipietro MA, Crawford J, Vanderhave $\mathrm{KL}$, Farley FA, et al. Serial ultrasound evaluation of pediatric trigger thumb. J Pediatr Orthop. 2013;33(3):309-13. 
Migoya-Nuño A, et al. Pulgar en gatillo

10. Masquijo JJ, Ferreyra A, Lanfranchi L, Torres-Gomez A, Allende V. Percutaneous trigger thumb release in children: Neither effective nor safe. J Pediatr Orthop. 2014;34(5):534-6.

11. Tan AHC, Lam KS, Lee EH. The treatment outcome of trigger thumb in children. J Pediatr Orthop Part B. 2002;11(3):256-9.

12. Han SH, Yoon HK, Shin DE, Song DG. Trigger thumb in children: Results of surgical treatment in children above 5 years of age. J Pediatr Orthop. 2010;30(7):710-4.

13. Sevencan A, Inan U, Köse N, Ömeroğlu H, Seber S. Percutaneous release for trigger thumbs in children: Improvements of the technique and results of 31 thumbs. J Pediatr Orthop. 2010;30(7):705-9.

14. Ruiz-Iban MA, Gonzalez-Herranz P, Mondejar JAL. Percutaneous trigger thumb release in children. J Pediatr Orthop. 2006;26(1):67-70.

15. Sirithiantong $T$, Woratanarat $P$, Woratanarat $T$, Angsanuntsukh C, Saisongcroh T, Unwanatham N, et al. Network meta-analysis of management of trigger thumb in children. J Pediatr Orthop B. 2020;Publish Ah:1-7.

16. Yano K, Ikeda M, Yoneda M, Tokui A, Nakagawa K, Kaneshiro $Y$, et al. Clinical results of splinting versus observation for pediatric trigger thumb. J Pediatr Orthop B. 2020;Publish Ah(1910082):8-12. 